\title{
DIFFERENTIATING-DOMINATING SETS IN GRAPHS UNDER BINARY OPERATIONS
}

\author{
SERGIO R. CANOY, JR. AND GINA A. MALACAS
}

\begin{abstract}
In this paper we characterize the differentiating-dominating sets in the join, corona, and lexicographic product of graphs. We also determine bounds or the exact differentiating-domination numbers of these graphs.
\end{abstract}

\section{Introduction}

Let $G=(V(G), E(G))$ be a connected graph and $v \in V(G)$. The neighborhood of $v$ is the set $N_{G}(\nu)=N(\nu)=\{u \in V(G): u v \in E(G)\}$. The degree of a vertex $v \in V(G)$ is equal to the cardinality of $N_{G}(\nu)$ and the maximum degree of $G$ is $\Delta(G)=\max \{\operatorname{deg}(\nu): v \in V(G)\}$.

If $X \subseteq V(G)$, then the open neighborhood of $X$ is the set $N_{G}(X)=N(X)=\cup_{v \in X} N_{G}(v)$. The closed neighborhood of $X$ is $N_{G}[X]=N[X]=X \cup N(X)$.

Now a connected graph $G$ of order $n \geq 3$ is point distinguishing if for any two distinct vertices $u$ and $v$ of $G, N_{G}[u] \neq N_{G}[v]$. It is totally point determining if for any two distinct vertices $u$ and $v$ of $G, N_{G}(u) \neq N_{G}(v)$ and $N_{G}[u] \neq N_{G}[v]$. These concepts were parts of investigation in [2] and [7].

A subset $X$ of $V(G)$ is a dominating set of $G$ if for every $v \in V(G) \backslash X$, there exists $x \in X$ such that $x v \in E(G)$, i.e., $N[X]=V(G)$. The domination number $\gamma(G)$ of $G$ is the smallest cardinality of a dominating set of $G$.

A subset $S$ of $V(G)$ is a locating set in a connected graph $G$ if for any two distinct vertices $u$ and $v$ in $V(G) \backslash S, N_{G}(u) \cap S \neq N_{G}(v) \cap S$. A subset $S$ of $V(G)$ is a differentiating set in a connected graph $G$ if for every two distinct vertices $u$ and $v$ of $G, N_{G}[u] \cap S \neq N_{G}[v] \cap S$. It is a strictly differentiating set if it is differentiating and $N_{G}[u] \cap S \neq S$ for all $u \in V(G)$. The

Received March 9, 2013, accepted April 9, 2014.

2010 Mathematics Subject Classification. 05C69.

Key words and phrases. Graph, dominating, differentiating, strictly differentiating, differentiatingdominating.

Corresponding author: Sergio R. Canoy, Jr.

Research (Research Project No. B-105) was funded by DOST-National Research Council of the Philippines. 
minimum cardinality of a differentiating set in $G$, denoted by $d n(G)$, is called the differentiating number of $G$. The minimum cardinality of a strictly differentiating set in $G$, denoted by $\operatorname{sdn}(G)$, is called the strict differentiating number of $G$. A differentiating (resp. strictly differentiating) subset $S$ of $V(G)$ which is also dominating is called a differentiating-dominating (resp. strictly differentiating-dominating) set in a graph $G$. The minimum cardinality of a differentiating-dominating (resp. strictly differentiating-dominating) set in $G$, denoted by $\gamma_{D}(G)$ (resp. $\gamma_{S D}(G)$ ), is called the differentiating-domination (resp. strict differentiatingdomination) number of $G$. Some of these concepts may be found in [4] and are investigated in [1], [3], [5], and [7].

In a given network or graph, a differentiating set can be viewed as a set of monitors which can actually determine the exact location of an intruder (e.g. a burglar, a fire, etc.). By requiring such a set to be dominating implies that every vertex where there is no monitor in it is connected to at least one monitoring device. Hence, determination of the differentiatingdomination number of a graph is equivalent to finding the least number of monitors that can do the certain task in a given graph or network. In some contexts, differentiating dominating sets are called identifying codes (see [8]).

Now let $G$ be a connected graph of order $n$ and suppose that there exist (distinct) adjacent vertices $u$ and $v$ of $G$ such that $N_{G}[u]=N_{G}[v]$. Then $N_{G}[u] \cap S=N_{G}[v] \cap S$ for any subset $S$ of $V(G)$. This implies that $G$ cannot have a differentiating set. Also, if $\Delta(G)=n-1$ and $v \in V(G)$ with $\operatorname{deg}(\nu)=n-1$, then $N_{G}[v] \cap S=S$ for any subset $S$ of $V(G)$. Consequently, $G$ cannot have a strictly differentiating set. Thus, unless otherwise stated, throughout this paper, $G$ is a point distinguishing graph of order $n \geq 3$. Moreover, whenever the concept of strictly differentiating set of a graph $G$ is mentioned in this paper, it is always assumed that $\Delta(G) \leq n-2$.

\section{Preliminary results and characterizations}

The following two simple observations are worth mentioning.

Remark 2.1. Every differentiating set in a connected graph $G$ is a locating set.

Remark 2.2. Let $G$ be a connected graph of order $n \geq 3$. Then $2 \leq \gamma_{D}(G) \leq n-1$.

Theorem 2.3. Let $G$ be a connected graph. Then $\gamma_{D}(G)=2$ if and only if $G=P_{3}$.

Proof. Suppose $\gamma_{D}(G)=2$, say $S=\{a, b\}$ is a differentiating dominating set in $G$. If $a b \in E(G)$, then $N_{G}[a] \cap S=\{a, b\}=N_{G}[b] \cap S$, contrary to our assumption of $S$. Therefore, $a b \notin E(G)$. Now, since $S$ has only three different non-empty subsets, $|V(G)|=3$. Therefore, since $G \neq K_{3}$, $G=P_{3}$.

For the converse, suppose that $G=[a, c, b]=P_{3}$. Let $S=\{a, b\}$. Then, clearly, $S$ is a differentiating dominating set in $G$. Thus $\gamma_{D}(G)=2$. 
Remark 2.4. Let $G$ be a connected graph of order $n \geq 3$. Then $d n(G) \leq \gamma_{D}(G) \leq \gamma_{S D}(G)$ and $d n(G) \leq \operatorname{sdn}(G) \leq \gamma_{S D}(G)$.

The following simple results give specific relationships between $d n(G), \operatorname{sdn}(G), \gamma_{D}(G)$, and $\gamma_{S D}(G)$ for a connected graph $G$.

Lemma 2.5. Let $G$ be a connected graph of order $n \geq 3$ such that $d n(G)<\gamma_{D}(G)$. Then $1+$ $\operatorname{dn}(G)=\gamma_{D}(G)$.

Proof. Let $S$ be a minimum differentiating set in $G$. Then $S$ is not a dominating set in $G$. Hence, there exists a $y \in V(G) \backslash S$ such that $x y \notin E(G)$ for all $x \in S$. This implies that $N_{G}[y] \cap$ $S=N_{G}(y) \cap S=\varnothing$. Set $S^{*}=S \cup\{y\}$ and let $z \in V(G) \backslash S^{*}$. Since $S$ is a locating set (Remark 2.1), $N_{G}(z) \cap S \neq \varnothing$. This implies that there exists $w \in S$ such that $w z \in E(G)$. This shows that $S^{*}$ is a dominating set in $G$. Next, let $a, b \in V(G)$. Then $N_{G}[a] \cap S \neq N_{G}[b] \cap S$ since $S$ is a differentiating set in $G$. Therefore, $N_{G}[a] \cap S^{*} \neq N_{G}[b] \cap S^{*}$. This implies that $S^{*}$ is a differentiating set in $G$. Therefore $\gamma_{D}(G) \leq 1+d n(G)$. Since $d n(G)<\gamma_{D}(G), 1+d n(G) \leq \gamma_{D}(G)$. This shows that $1+d n(G)=\gamma_{D}(G)$.

Lemma 2.6. Let $G$ be a connected graph of order $n \geq 3$ such that $d n(G)<\operatorname{sdn}(G)$ and $\Delta(G) \leq$ $n-2$. Then $1+d n(G)=\operatorname{sdn}(G)$.

Proof. Let $S$ be a minimum differentiating set in $G$. By assumption, $S$ is not a strictly differentiating set in $G$. Hence, there exists a $y \in V(G)$ such that $N_{G}[y] \cap S=S$. Since $\operatorname{deg}(y) \leq n-2$, there exists $z \in V(G) \backslash(S \cup\{y\})$ such that $z \notin N_{G}(y)$. Set $S^{*}=S \cup\{z\}$. If $a, b \in V(G)(a \neq b)$, then $N_{G}[a] \cap S \neq N_{G}[b] \cap S$ since $S$ is a differentiating set. Thus, $N_{G}[a] \cap S^{*} \neq N_{G}[b] \cap S^{*}$, showing that $S^{*}$ is a differentiating set. Now let $x \in V(G)$. If $x=y$, then $z \notin N_{G}[x]$. This implies that $z \notin N_{G}[x] \cap S^{*}$. Hence $N_{G}[x] \cap S^{*} \neq S^{*}$. If $x \neq y$, then $N_{G}[x] \cap S \neq S$ since $S$ is differentiating. This implies that there exists $w \in S$ such that $w \notin N_{G}[x]$. Hence, $N_{G}[x] \cap S^{*} \neq S^{*}$. Therefore $S^{*}$ is a strictly differentiating set in $G$. Consequently, $s d n(G) \leq 1+d n(G)$. Since $d n(G)<s d n(G)$, $1+d n(G) \leq \operatorname{sdn}(G)$. This establishes the desired equality.

Lemma 2.7. Let $G$ be a connected graph of order $n \geq 3$ such that $\operatorname{sdn}(G)<\gamma_{S D}(G)$. Then $1+\operatorname{sdn}(G)=\gamma_{S D}(G)$.

Proof. Let $S$ be a minimum strictly differentiating set in $G$. From the assumption, $S$ is not a dominating set in $G$. Hence, there exists a $y \in V(G) \backslash S$ such that $x y \notin E(G)$ for all $x \in S$. This implies that $N_{G}[y] \cap S=N_{G}(y) \cap S=\varnothing$. Set $S^{*}=S \cup\{y\}$ and let $z \in V(G) \backslash S^{*}$. Since $S$ is a differentiating set, $N_{G}[z] \cap S=N_{G}(z) \cap S \neq \varnothing$. This implies that there exists $q \in S \subseteq S^{*}$ such that $q z \in E(G)$. Hence $S^{*}$ is a dominating set in $G$. 
Next, let $a, b \in V(G)$. Then $N_{G}[a] \cap S \neq N_{G}[b] \cap S$ since $S$ is a differentiating set in $G$. Therefore, $N_{G}[a] \cap S^{*} \neq N_{G}[b] \cap S^{*}$. This implies that $S^{*}$ is a differentiating set in $G$. Moreover, if $x \in V(G)$, then $N_{G}[x] \cap S \neq S$ since $S$ is strictly differentiating. It follows that $N_{G}[x] \cap S^{*} \neq S^{*}$, i.e., $S^{*}$ is a strictly differentiating (dominating) set. Therefore $\gamma_{S D}(G) \leq 1+\operatorname{sdn}(G)$. Since $s d n(G)<\gamma_{S D}(G), 1+s d n(G) \leq \gamma_{S D}(G)$. This shows that $1+s d n(G)=\gamma_{S D}(G)$.

Lemma 2.8. Let $G$ be a connected graph of order $n \geq 3$ such that $\gamma_{D}(G)<\gamma_{S D}(G)$. Then $1+$ $\gamma_{D}(G)=\gamma_{S D}(G)$.

Proof. Let $S$ be a minimum differentiating dominating set in $G$. Then $S$ is not a strictly differentiating set in $G$. Hence, there exists a $y \in V(G)$ such that $N_{G}[y] \cap S=S$. Since $\operatorname{deg}(y) \leq n-2$, there exists $z \in V(G) \backslash(S \cup\{y\})$ such that $z \notin N_{G}(y)$. Set $S^{*}=S \cup\{z\}$. Since $S$ is a dominating set, $S^{*}$ is also a dominating set. If $a, b \in V(G)$, then $N_{G}[a] \cap S \neq N_{G}[b] \cap S$ since $S$ is a differentiating set. Thus, $N_{G}[a] \cap S^{*} \neq N_{G}[b] \cap S^{*}$, showing that $S^{*}$ is a differentiating set. Now let $x \in V(G)$. If $x=y$, then $z \notin N_{G}[x]$. This implies that $z \notin N_{G}[x] \cap S^{*}$. Hence $N_{G}[x] \cap S^{*} \neq S^{*}$. If $x \neq y$, then $N_{G}[x] \cap S \neq S$ since $S$ is differentiating. This implies that there exists $w \in S$ such that $w \notin N_{G}[x]$. It follows that $N_{G}[x] \cap S^{*} \neq S^{*}$. Therefore $S^{*}$ is a strictly differentiating (dominating) set in $G$. Consequently, $\gamma_{S D}(G) \leq 1+\gamma_{D}(G)$. Since $\gamma_{D}(G)<\gamma_{S D}(G), 1+\gamma_{D}(G) \leq \gamma_{S D}(G)$. Accordingly, $1+\gamma_{D}(G)=\gamma_{S D}(G)$.

\section{Differentiating dominating sets in the join of graphs}

The join $G+H$ of two graphs $G$ and $H$ is the graph with $V(G+H)=V(G) \cup V(H)$ (disjoint union) and $E(G+H)=E(G) \cup E(H) \cup\{u v: u \in V(G)$ and $v \in V(H)\}$.

Theorem 3.9. Let $G$ and $H$ be non-trivial graphs of orders $m \geq 2$ and $n \geq 2$, respectively. Then $S \subseteq V(G+H)$ is a differentiating dominating set in $G+H$ if and only if $S_{G}=V(G) \cap S$ and $S_{H}=V(H) \cap S$ are differentiating sets in $G$ and $H$, respectively, and either $S_{G}$ or $S_{H}$ is strictly differentiating.

Proof. Let $S \subseteq V(G+H)$ be a differentiating-dominating set in $G+H$. Let $S_{G}=V(G) \cap S$ and $S_{H}=V(H) \cap S$. Suppose $S_{G}=\varnothing$. Pick distinct vertices $u$ and $v$ of $G$. Then $N_{G+H}[u] \cap S=$ $S=N_{G+H}[v] \cap S$, contrary to the assumption that $S$ is a differentiating set for $G+H$. Thus, $S_{G} \neq \varnothing$. Similarly, $S_{H} \neq \varnothing$. Suppose now that one of $S_{G}$ and $S_{H}$ is not a differentiating set, say $S_{G}$ is not a differentiating set in $G$. Then there exist distinct vertices $a, b \in V(G)$ such that $N_{G}[a] \cap S_{G}=N_{G}[b] \cap S_{G}$. Since $S_{H} \subseteq N_{G+H}[a]$ and $S_{H} \subseteq N_{G+H}[b]$, it follows that $N_{G+H}[a] \cap S=$ $\left(N_{G}[a] \cap S_{G}\right) \cup S_{H}=N_{G+H}[b] \cap S$. This is impossible since $S$ is a differentiating set for $G+H$. Therefore, $S_{G}$ and $S_{H}$ are differentiating sets in $G$ and $H$, respectively.

Next, suppose that both $S_{G}$ and $S_{H}$ are not strictly differentiating sets in $G$ and $H$, respectively. Then there exist $z \in V(G) \backslash S_{G}$ and $w \in V(H) \backslash S_{H}$ such that $N_{G}[z] \cap S_{G}=S_{G}$ and 
$N_{H}[w] \cap S_{H}=S_{H}$. It follows that $N_{G+H}[z] \cap S=S=N_{G+H}[w] \cap S$, contrary to the fact that $S$ a differentiating set in $G+H$. Thus, $S_{G}$ is a strictly differentiating set in $G$ or $S_{H}$ is a strictly differentiating set in $H$.

For the converse, suppose $S_{G}=V(G) \cap S$ and $S_{H}=V(H) \cap S$ are differentiating sets in $G$ and $H$, respectively, and where $S_{G}$ or $S_{H}$ is a strictly differentiating set. Let $x$ and $y$ be distinct vertices in $V(G+H)$. If $x, y \in V(G)$, then $N_{G}[x] \cap S_{G} \neq N_{G}[y] \cap S_{G}$. It follows that $N_{G+H}[x] \cap S=$ $\left(N_{G}[x] \cap S_{G}\right) \cup S_{H} \neq\left(N_{G}[y] \cap S_{G}\right) \cup S_{H}=N_{G+H}[y] \cap S$. Similarly, $N_{G+H}[x] \cap S \neq N_{G+H}[y] \cap S$ if $x, y \in V(H)$. Suppose $x \in V(G)$ and $y \in V(H)$. Suppose, without loss of generality, that $S_{G}$ is a strictly differentiating set in $G$. Then $S_{G}$ is not contained in $N_{G+H}[x]$. Since $S_{G} \subseteq N_{G+H}[y]$, it follows that $N_{G+H}[x] \cap S \neq N_{G+H}[y] \cap S$. Accordingly, $S$ is a differentiating set in $G+H$. Clearly, $S$ is a dominating set in $G+H$.

The next results are direct consequences of Theorem 3.9 or of its proof.

Corollary 3.10. Let $G$ and $H$ be connected non-trivial graphs of orders $m \geq 2$ and $n \geq 2$, respectively. Then every differentiating set in $G+H$ is dominating.

Corollary 3.11. Let $G$ and $H$ be connected non-trivial graphs of orders $m \geq 2$ and $n \geq 2$, respectively. Then $\gamma_{D}(G+H)=\min \{s d n(H)+d n(G), s d n(G)+d n(H)\}$.

Theorem 3.12. Let $G=K_{1}=\langle\nu\rangle$ and $H$ a non-trivial graph. Then $S \subseteq V(G+H)$ is a differentiating dominating set in $G+H$ if and only if $v \in S$ and $V(H) \cap S$ is a strictly differentiating set in $H$ or $v \notin S$ and $S$ is a strictly differentiating dominating set in $H$.

Proof. Suppose $S$ is a differentiating dominating set in $G+H$ and suppose $v \in S$. Since $S$ is differentiating and $|V(H)| \geq 2, V(H) \cap S \neq \varnothing$. Also, since $N_{G+H}[v] \cap S=S, V(H) \cap S$ must be a strictly differentiating set in $H$. Suppose now that $v \notin S$. Then $S \subseteq V(H)$ must be a dominating set in $H$. Since $N_{G+H}[u] \cap S=N_{H}[u] \cap S$ for every $u \in V(H)$ and $N_{G+H}[v] \cap S=S, S$ is a strictly differentiating set in $H$. Hence $S$ is a strictly differentiating dominating set in $H$.

The converse is clear.

Corollary 3.13. Let $G=K_{1}=\langle\nu\rangle$ and $H$ a non-trivial graph. Then $\gamma_{D}(G+H)=\gamma_{S D}(H)$.

Proof. Let $S$ be a minimum differentiating dominating set in $G+H$. Suppose first that $v \in S$. Then $V(H) \cap S$ is a strictly differentiating set in $H$, by Theorem 3.12. Hence, $\operatorname{sdn}(G)+1 \leq|S|=$ $\gamma_{D}(G+H)$. By Remark 2.4 and Lemma 2.7, $\gamma_{S D}(H) \leq \gamma_{D}(G+H)$. If $v \notin S$, then $S$ is a strictly differentiating dominating set in $H$ by Theorem 3.12. It follows that $\gamma_{S D}(H) \leq|S|=\gamma_{D}(G+H)$. Thus $\gamma_{D}(G+H) \geq \gamma_{S D}(H)$.

Now let $S$ be a minimum strictly differentiating dominating set in $H$. Then $S$ is a differentiating dominating set in $G+H$ by Theorem 3.12. Thus $\gamma_{D}(G+H) \leq|S|=\gamma_{S D}(H)$. 
Therefore $\gamma_{D}(G+H)=\gamma_{S D}(H)$.

\section{Differentiating dominating sets in the corona of graphs}

The corona $G \circ H$ of two graphs $G$ and $H$ is the graph obtained by taking one copy of $G$ of order $n$ and $n$ copies of $H$, and then joining the $i^{\text {th }}$ vertex of $G$ to every vertex in the $i^{\text {th }}$ copy of $H$. For every $v \in V(G)$, we denote by $H^{v}$ the copy of $H$ whose vertices are attached one by one to the vertex $v$. Subsequently, we denote by $v+H^{v}$ the subgraph of the corona $G \circ H$ corresponding to the join $\langle\nu\rangle+H^{v}$, where $v \in V(G)$.

Theorem 4.14. Let $G$ (not necessarily point distinguishing) and let $H$ be non-trivial connected graphs. Then $C \subseteq V(G \circ H)$ is a differentiating dominating set in $G \circ H$ if and only if for every $v \in V(G)$, one of the following is true:

(i) $v \in C, N_{G}(v) \cap C \neq \varnothing$, and $C \cap V\left(H^{v}\right)$ is a differentiating set in $H^{v}$;

(ii) $v \in C, N_{G}(v) \cap C=\varnothing$, and $C \cap V\left(H^{v}\right)$ is a strictly differentiating set in $H^{v}$;

(iii) $v \notin C, N_{G}(v) \cap C \neq \varnothing$, and $C_{1}=V\left(H^{v}\right) \cap C$ is a differentiating dominating set in $H^{v}$; or

(iv) $v \notin C, N_{G}(v) \cap C=\varnothing$ and $C_{1}=V\left(H^{v}\right) \cap C$ is a strictly differentiating dominating set in $H^{v}$.

Proof. Suppose $C$ is a differentiating dominating set in $G \circ H$. Let $v \in V(G), C_{1}=V\left(H^{v}\right) \cap C$, and let $x, y \in V\left(H^{v}\right)$, where $x \neq y$. Then $N_{G \circ H}[x] \cap C=\left(N_{H^{v}}[x] \cap C_{1}\right) \cup(C \cap\{v\}), N_{G \circ H}[y] \cap C=$ $\left(N_{H^{v}}[y] \cap C_{1}\right) \cup(C \cap\{v\})$, and $N_{G \circ H}[v] \cap C=\left(N_{G}(\nu) \cap C_{2}\right) \cup\left(N_{v+H^{v}}[v] \cap C_{1}\right) \cup(C \cap\{v\})$, where $C_{2}=C \cap V(G)$.

Suppose first that $v \in C$. If $N_{G}(v) \cap C \neq \varnothing$, then, since $C$ is differentiating,

$$
\left(N_{H^{v}}[x] \cap C_{1}\right) \cup\{v\}=N_{G \circ H}[x] \cap C \neq N_{G \circ H}[y] \cap C=\left(N_{H^{v}}[y] \cap C_{1}\right) \cup\{v\} .
$$

It folows that $N_{H^{v}}[x] \cap C_{1} \neq N_{H^{v}}[y] \cap C_{1}$. Thus $C_{1}$ is differentiating in $H^{v}$, i.e., (i) holds. Suppose $N_{G}(v) \cap C=\varnothing$. Then, again, since $C$ is differentiating, $C_{1}$ is differentiating in $H^{v}$. Now, since $N_{G \circ H}[v] \cap C=N_{\nu+H^{v}}[v] \cap C_{1}=\{\nu\} \cup C_{1}, C_{1}$ must be strictly differentiating in $H^{v}$. Hence, (ii) holds.

Next, suppose that $v \notin C$. If $N_{G}(v) \cap C \neq \varnothing$, then, since $C$ is differentiating,

$$
N_{H^{v}}[x] \cap C_{1}=N_{G \circ H}[x] \cap C \neq N_{G \circ H}[y] \cap C=N_{H^{v}}[y] \cap C_{1} .
$$

This implies that $C_{1}$ is differentiating in $H^{v}$. Since $v \notin C$ and $C$ is dominating, $C_{1}$ is dominating in $H^{v}$. Therefore, (iii) holds. Suppose $N_{G}(v) \cap C=\varnothing$. Since $C$ is a differentiating dominating set, $v \notin C$, and $N_{G \circ H}[v]=C_{1}$, it follows that $C_{1}$ is a strictly differentiating dominating set in $H^{v}$, i.e., (iv) holds.

For the converse, suppose that $C$ satisfies (i), (ii), (iii), or (iv) for every $v \in V(G)$. Let $x \in V(G \circ H) \backslash C$ and $v \in V(G)$ be such that $x \in V\left(v+H^{v}\right)$. If $v \in C$, then $x v \in E(G \circ H)(x \neq v)$. If 
$v \notin C$ (say when $x=v$ ), then, by (iii), or (iv), $C_{1}=V\left(H^{v}\right) \cap C$ is a dominating set in $H^{v}$. Hence, there exists $y \in C_{1}$ such that $x y \in E\left(H^{v}\right) \subseteq E(G \circ H)$. Therefore, $C$ is a dominating set in $G \circ H$.

Next, let $a, b \in V(G \circ H)$ with $a \neq b$. Let $u, v \in V(G)$ such that $a \in V\left(u+H^{u}\right)$ and $b \in$ $V\left(v+H^{v}\right)$. Consider the following cases:

Case 1: Suppose that $u=v$.

If $a, b \in V\left(H^{v}\right)$, then $N_{H^{v}}[a] \cap C_{1} \neq N_{H^{v}}[b] \cap C_{1}$ since $C_{1}$ is differentiating in $H^{v}$ by (i), (ii), (iii), and (iv). Therefore,

$$
\left(N_{G \circ H}[a] \cap C\right) \cup(\{v\} \cap C) \neq\left(N_{G \circ H}[b] \cap C\right) \cup(\{v\} \cap C) \text {, i.e., }
$$

$N_{G \circ H}[a] \cap C \neq N_{G \circ H}[b] \cap C$.

Suppose $a=v$ and $b \in V\left(H^{\nu}\right)$. If $N_{G}(v) \cap C \neq \varnothing$, say $z \in N_{G}(v) \cap C$, then $z \in\left[N_{G \circ H}[a] \cap\right.$ $C] \backslash\left[N_{G \circ H}[b] \cap C\right]$. Thus, $N_{G \circ H}[a] \cap C \neq N_{G \circ H}[b] \cap C$. If $N_{G}(v) \cap C=\varnothing$, then $V\left(H^{\nu}\right) \cap C$ is strictly differentiating in $H^{v}$ by (ii) and (iv). Hence, there exists $w \in V\left(H^{\nu}\right) \cap C$ such that $w \notin N_{G \circ H}[b] \cap C$. Since $w \in N_{G \circ H}[a] \cap C$, it follows that $N_{G \circ H}[a] \cap C \neq N_{G \circ H}[b] \cap C$.

Case 2: Suppose that $u \neq v$.

Since $V\left(H^{u}\right) \cap C$ and $V\left(H^{v}\right) \cap C$ are non-empty disjoint sets, and $V\left(H^{u}\right) \cap C \cap N_{G \circ H}[a] \cap C \neq$ $\varnothing$ and $V\left(H^{\nu}\right) \cap C \cap N_{G \circ H}[b] \cap C \neq \varnothing$, it follows that $N_{G \circ H}[a] \cap C \neq N_{G \circ H}[b] \cap C$.

Accordingly, $C$ is a differentiating dominating set in $G \circ H$.

Corollary 4.15. Let $G$ (not necessarily point distinguishing) and let $H$ (point distinguishing) be non-trivial connected graphs. Then

$$
|V(G)| \gamma_{D}(H) \leq \gamma_{D}(G \circ H) \leq|V(G)| \gamma_{S D}(H) .
$$

Proof. Let $C$ be a minimum differentiating dominating set in $G$. Then

$$
\gamma_{D}(G \circ H)=|C|=\sum_{\nu \in V(G) \cap C}\left(1+\left|V\left(H^{\nu}\right) \cap C\right|\right)+\sum_{\nu \in V(G) \backslash C}\left|V\left(H^{\nu}\right) \cap C\right| .
$$

From Theorem 4.14(i) and (ii), Remark 2.4, and Lemma 2.5, $1+\left|V\left(H^{\nu}\right) \cap C\right| \geq 1+d n(H) \geq$ $\gamma_{D}(H)$ for every $v \in V(G) \cap C$. Now, if $v \in V(G) \backslash C$ and $N_{G}(v) \cap C \neq \varnothing$, then $\left|V\left(H^{v}\right) \cap C\right| \geq \gamma_{D}(H)$ by Theorem 4.14(iii). If $N_{G}(v) \cap C=\varnothing$, then $\left|V\left(H^{v}\right) \cap C\right| \geq \gamma_{S D}(H) \geq \gamma_{D}(H)$ by Theorem 4.14(iv) and Remark 2.4. Thus, $\left|V\left(H^{\nu}\right) \cap C\right| \geq \gamma_{D}(H)$ for every $v \in V(G) \backslash C$. Therefore $\gamma_{D}(G \circ H)=|C| \geq$ $|V(G)| \gamma_{D}(H)$.

Next, let $S$ be a minimum strictly differentiating-dominating set in $H$. For each $v \in V(G)$, pick $S_{\nu} \subseteq V\left(H^{v}\right)$, where $\left\langle S_{\nu}\right\rangle \cong\langle S\rangle$. Then $C=\cup_{v \in V(G)} S_{\nu}$ is a differentiating dominating set in $G \circ H$ by Theorem 4.14. Hence, $\gamma_{D}(G \circ H) \leq|C|=|V(G)| \gamma_{S D}(H)$.

Therefore, $|V(G)| \gamma_{D}(H) \leq \gamma_{D}(G \circ H) \leq|V(G)| \gamma_{S D}(H)$. 


\section{Differentiating dominating sets in the lexicographic product of graphs}

The lexicographic product $G[H]$ of two graphs $G$ and $H$ is the graph with $V(G[H])=$ $V(G) \times V(H)$ and $\left(u, u^{\prime}\right)\left(\nu, v^{\prime}\right) \in E(G[H])$ if and only if either $u v \in E(G)$ or $u=v$ and $u^{\prime} v^{\prime} \in E(H)$.

Observe that any subset $C$ of $V(G) \times V(H)$ (in fact, any set of ordered-pairs) can be written as $C=\cup_{x \in S}\left(\{x\} \times T_{x}\right)$, where $S \subseteq V(G)$ and $T_{x} \subseteq V(H)$ for each $x \in S$. Henceforth, we shall use this form to denote any subset $C$ of $V(G) \times V(H)$.

Theorem 5.16. Let $G$ (not necessarily point distinguishing) and $H$ be non-trivial connected graphs. Then $C=\cup_{x \in S}\left(\{x\} \times T_{x}\right)$, where $S \subseteq V(G)$ and $T_{x} \subseteq V(H)$ for each $x \in S$, is a differentiatingdominating set in $G[H]$ if and only if

(i) $S=V(G)$;

(ii) $T_{x}$ is a differentiating set in $H$ for every $x \in V(G)$;

(iii) $T_{x}$ or $T_{y}$ is strictly differentiating in $H$ whenever $x$ and $y$ are adjacent vertices of $G$ with $N_{G}[x]=N_{G}[y] ;$ and

(iv) $T_{x}$ or $T_{y}$ is (differentiating) dominating in $H$ whenever $x$ and $y$ are distinct non-adjacent vertices of $G$ with $N_{G}(x)=N_{G}(y)$.

Proof. Suppose $C$ is a differentiating dominating set in $G[H]$. Suppose there exists $x \in V(G) \backslash S$. Pick $a, b \in V(H)$, where $a \neq b$. Then $(x, a),(x, b) \notin C$ and $(x, a) \neq(x, b)$. Since $\{(x, c): c \in$ $V(H)\} \cap C=\varnothing$, it follows that $N_{G[H]}[(x, a)] \cap C=N_{G[H]}[(x, b)] \cap C$. This implies that $C$ is not a differentiating set in $G[H]$, contrary to our assumption. Therefore, $S=V(G)$.

Now let $x \in V(G)$ and suppose that $T_{x}$ is not differentiating in $H$. Then there exists distinct vertices $p$ and $q$ in $V(H)$ such that $N_{H}[p] \cap T_{x}=N_{H}[q] \cap T_{x}$. Let $D_{x}=N_{H}[p] \cap T_{x}$. Since $N_{G[H]}[(x, p)] \cap C=\cup\left\{\{y\} \times T_{y}: y \in N_{G}(x)\right\} \cup\left(\{x\} \times D_{x}\right)=N_{G[H]}[(x, q)] \cap C$, it follows that $C$ is a not a differentiating set in $G[H]$. Again, this gives a contradiction. Therefore, $T_{x}$ is a differentiating set in $H$.

To prove (iii), let $x$ and $y$ be adjacent vertices of $G$ with $N_{G}[x]=N_{G}[y]$. Suppose that $T_{x}$ and $T_{y}$ are not strictly differentiating in $H$. Then there exist $c, d \in V(H)$ such that $N_{H}[c] \cap T_{x}=$ $T_{x}$ and $N_{H}[d] \cap T_{y}=T_{y}$. It follows that $\left(\{x\} \times T_{x}\right) \cup\left(\{y\} \times T_{y}\right) \subseteq N_{G[H]}[(x, c)] \cap N_{G[H]}[(y, d)]$. Since $N_{G}[x]=N_{G}[y]$, it follows that $N_{G[H]}[(x, c)] \cap C=N_{G[H]}[(y, d)] \cap C$, i.e., $C$ is not a differentiating set in $G[H]$. This contradicts our assumption. Therefore, $T_{x}$ or $T_{y}$ is strictly differentiating in $H$.

To prove (iv), let $x$ and $y$ be distinct non-adjacent vertices of $G$ with $N_{G}(x)=N_{G}(y)$. Suppose that $T_{x}$ is not a dominating set in $H$. Then there exists $a \in V(H) \backslash T_{x}$ such that $a b \notin E(H)$ for all $b \in T_{x}$. It follows that $(x, a) \notin C$ and $N_{G[H]}[(x, a)] \cap C=N_{G[H]}((x, a)) \cap C=$ $\cup\left\{\{z\} \times T_{z}: z \in N_{G}(x)\right\}$. Let $c \in V(H) \backslash T_{y}$. Then $(y, c) \notin C$. Since $N_{G}(x)=N_{G}(y)$, it follows that 
$\cup\left\{\{z\} \times T_{z}: z \in N_{G}(x)\right\} \subseteq N_{G[H]}((y, c)) \cap C=N_{G[H]}[(y, c)] \cap C$. Since $C$ is a differentiating set in $G[H]$, there exists $(y, d) \in\{y\} \times T_{y}$ such that $(y, d)(y, c) \in E(G[H])$. This implies that $d \in T_{y}$ and $c d \in E(H)$. Therefore, $T_{y}$ is a dominating set in $H$. This shows that (iv) holds.

For the converse, suppose that conditions (i), (ii), (iii), and (iv) hold. By (i) and the fact that $G$ is connected, it follows that $C$ is a dominating set in $G[H]$. Now let $(x, a),(y, b) \in$ $V(G[H])$ with $(x, a) \neq(y, b)$. Consider the following cases:

Case 1. Suppose $x=y$.

Then $a \neq b$. Since $T_{x}$ is a differentiating set in $H, N_{H}[a] \cap T_{x}=A \neq B=N_{H}[b] \cap T_{y}$. Now, since $\left(\{x\} \times T_{x}\right) \cap\left(N_{G[H]}[(x, a)] \cap C\right)=\{x\} \times A$ and $\left(\{y\} \times T_{y}\right) \cap\left(N_{G[H]}[(y, b)] \cap C\right)=\{y\} \times B$, it follows that $N_{G[H]}[(x, a)] \cap C \neq N_{G[H]}[(y, b)] \cap C$.

Case 2. Suppose $x \neq y$.

Consider the following sub-cases:

Sub-case 1 . Suppose $x y \notin E(G)$.

Suppose first that $N_{G}(x) \neq N_{G}(y)$, say $z \in N_{G}(x) \backslash N_{G}(y)$. Pick $d \in T_{z}$. Then $(z, d) \in C$ and $(z, d) \in\left(N_{G[H]}[(x, a)] \cap C\right) \backslash\left(N_{G[H]}[(y, b)] \cap C\right)$. Next, suppose that $N_{G}(x)=N_{G}(y)$. By (iv), we may assume that $T_{x}$ is a dominating set in $H$. If $(x, a) \in C$, then $(x, a) \in\left(N_{G[H]}[(x, a)] \cap\right.$ $C) \backslash\left(N_{G[H]}[(y, b)] \cap C\right)$. If $(x, a) \notin C$, then $a \notin T_{x}$. Hence, there exists $c \in T_{x}$ such that $a c \in E(H)$. This implies that $(x, c) \in C$ and $(x, c) \in\left(N_{G[H]}[(x, a)] \cap C\right) \backslash\left(N_{G[H]}[(y, b)] \cap C\right)$.

Sub-case 2. Suppose $x y \in E(G)$.

If $N_{G}[x] \neq N_{G}[y]$, then $N_{G}(x) \neq N_{G}(y)$; hence, as in a previous case, $N_{G[H]}[(x, a)] \cap C \neq$ $N_{G[H]}[(y, b)] \cap C$. If $N_{G}[x]=N_{G}[y]$, then, by (iii), it can be assumed that $T_{x}$ is strictly differentiating in $H$. Hence $N_{H}[a] \cap T_{x} \neq T_{x}$. This implies that there exists $q \in T_{x}$ such that $q \notin N_{H}[a]$. It follows that $(x, q) \in C$ and $(x, q) \in\left(N_{G[H]}[(y, b)] \cap C\right) \backslash\left(N_{G[H]}[(x, a)] \cap C\right)$.

Accordingly, $C$ is a differentiating-dominating set in $G[H]$.

The following is a direct consequence of Theorem 5.16.

Corollary 5.17. Let $G$ be a non-trivial connected totally point determining graph and $H$ a nontrivial connected point distinguishing graph with $\Delta(H) \leq|V(H)|-2$. Then $C=\cup_{x \in S}\left(\{x\} \times T_{x}\right)$, where $S \subseteq V(G)$ and $T_{x} \subseteq V(H)$ for each $x \in S$, is a minimum differentiating-dominating set in $G[H]$ if and only if $S=V(G)$ and each $T_{x}$ is a minimum differentiating set in $H$.

The next result is immediate from Corollary 5.17 .

Corollary 5.18. Let $G$ be a non-trivial connected totally point determining graph and $H$ a non-trivial connected point distinguishing graph with $\Delta(H) \leq|V(H)|-2$. Then $\gamma_{D}(G[H])=$ $|V(G)| \gamma_{D}(H)$. 
Proof. Let $C=\cup_{x \in S}\left(\{x\} \times T_{x}\right)$ be a minimum differentiating dominating set in $G[H]$. Then $S=V(G)$ and $T_{x}$ is a minimum differentiating set in $H$ for every $x \in V(G)$, by Corollary 5.17. It follows that $\gamma_{D}(G[H])=|C|=|V(G)| \operatorname{dn}(H)$.

\section{Acknowledgement}

The authors are very grateful to the referee for pointing out errors in the original manuscript and for giving invaluable suggestions which led to this much improved version of the paper.

\section{References}

[1] M. Frick, C. M. Mynhardt and R. D Skaggs, Critical graphs with respect to vertex identification, Utilitas Math., 76 (2008), 213-227.

[2] D. Geoffroy, Nuclei for totally point distinguishing graphs, Discrete Math., 21(1978),145-162.

[3] J. Gimbel, B. D. van Gorden, M. Nicolescu, C. Umstead, and N. Vaiana, Location with dominating sets, Congr. Numer., 151(2001), 129-144.

[4] T. W. Haynes, M. A. Henning, and J. Howard, Locating and total dominating sets in trees, Discrete Applied Mathematics, 154(2006), Issue 8, 1293-1300.

[5] T. W. Haynes, S. T. Hedetniemi, and P. J. Slater, Fundamentals of Domination in Graphs. Marcel Dekker, New York, 1998.

[6] M. A. Henning, John McCoy, Which trees have a differentiating-paired dominating set? Journal of Combinatorial Optimization, 22(2011), Number 1, 1-18.

[7] D. P. Sumner, Point determination in graphs, Discrete Math., 5(1973),179-187.

[8] M. G. Karpovsky, K. Chakrabarty, and L. B. Levitin, On a new class of codes for identifying vertices in graphs, IEEE Trans. Inform. Theory, 44(2) (1998), 599-611.

Department of Mathematics and Statistics, College of Science and Mathematics, Mindanao State University Iligan Institute of Technology, 9200 Iligan City, Philippines.

E-mail: serge_canoy@yahoo.com

Department of Mathematics and Statistics, College of Science and Mathematics, Mindanao State University Iligan Institute of Technology, 9200 Iligan City, Philippines.

E-mail: ginastrong@math.com 